\title{
La promoción de la lectura y el trasvase cultural literario: una investigación en Enseñanza Secundaria Obligatoria basada en el tópico del amor
}

The promotion of reading and the transfer of literary culture: an investigation in secondary school education based on the topic of love

\author{
EDUARDO ENCABO FERNÁNDEZ \\ ANDRÉS MONTANER BUENO \\ Universidad de Murcia \\ España \\ edencabo@um.es \\ andres_5rayos@hotmail.com
}

Resumen. El presente artículo constituye una investigación realizada en el marco de la didáctica de la lengua y la literatura con el objetivo de llevar a cabo la promoción de la lectura a través del tópico literario del amor. Para ello, nos hemos servido de tres textos de referencia de distintas épocas: El mito de Píramo y Tisbe (Antigüedad Clásica), Romeo y Julieta (Siglo XVI) y A tres metros sobre el cielo (Siglo XXI).A través de la comparación de los mismos $y$, mediante el empleo de una serie de instrumentos de medida, hemos tratado de potenciar en el alumnado de cuarto de Educación Secundaria Obligatoria del IES Amparo Sanz de Albacete, la capacidad crítica y reflexiva a la hora de acometer el acto de lectura. Para llevar a cabo este objetivo, nos hemos servido de las múltiples posibilidades y sugerencias que nos ofrece el intertexto lector.

Palabras clave: Promoción de la lectura;Tópicos literarios; Lecturas canónicas; Intertexto lector.
Abstract. This article is an investigation conducted, within the framework of Spanish language and literature teaching, with the aim of promoting reading through the literary topic of love. To this aim, we have used three reference texts written in different eras: Pyramus and Thisbe myth (Classical Antiquity), Romeo and Juliet (sixteenth century) and Something's Triggered (twenty-first century). Through the comparison of them, using a range of measuring instruments, we have tried to enhance the student's critical and reflective capacity when they undertake the act of read-ing. To accomplish this goal, we have used the several possibilities and suggestions offered by the reader intertext. The students were in the 4th E.S.O level at Amparo Sanz highschool, located in Albacete.

Key words: Promote reading; Literary topics; Reading canonical; Intertexto reader

\footnotetext{
I Para citar este artículo: Encabo Fernández, E. y Montaner Bueno, A. La promoción de la lectura y el trasvase cultural literario: una investigación en Enseñanza Secundaria Obligatoria basada en el tópico del amor. Alabe 5, junio 2012

[http://www.ual.es/alabe]
} 


\section{Introducción}

Considerando que la lectura es una de las cinco habilidades básicas que contribuyen a desarrollar la competencia comunicativa de una persona -junto a hablar, escuchar, conversar y escribir-, se entenderá en seguida la importancia de este tema en el sistema en el que vivimos. Leer constituye una actividad básica para una formación completa del ser humano ya que permite reflexionar sobre diferentes temas y desarrollar un pensamiento crítico. Esta necesidad se ve acrecentada en la actual sociedad de la información en la que tenemos que estar continuamente descifrando e interpretando múltiples y variados mensajes.

Así pues, entendemos la lectura como una actividad dinámica en la que el sujeto y el texto interactúan. Asimismo, a través del acto de leer se ponen en funcionamiento una serie de procesos cognitivos que activan los conocimientos previos de los sujetos lectores, que van a actuar como marco de referencia. Desde una perspectiva fenomenológica, en la consideración de la lectura se ha de valorar no solamente el texto sino también los actos de recepción. De esta situación se deducen dos polos: uno artístico y otro estético, siendo el artístico el texto creado por el autor, y el estético la concreción realizada por el lector. El lugar de la obra de arte es la convergencia de texto y lector, y posee forzosamente carácter virtual, puesto que no puede reducirse ni a la realidad del texto ni a las disposiciones que constituyen al lector. La obra adquiere su auténtico carácter procesal únicamente en el proceso de su lectura.

Según W. Iser (1987) y Jauss (I977) es en el texto donde el autor comparte sus asuntos con el lector dejándole que imagine también algo. Esto es muy importante ya que el lector sólo obtiene satisfacción cuando pone en juego su productividad, y ello sólo ocurre cuando el texto ofrece la posibilidad de ejercitar sus capacidades a través del intertexto de la propia persona. En los textos, además, lo que se dice aparece ante un trasfondo que actúa significativamente dejando adivinar algunos datos. Surge un espacio de sugestiones a través de las cuales, determinadas escenas triviales adquieren un carácter significativo.

De esta teoría sobre el proceso de lectura, se deduce que la misma se nos revela como un factor determinante en la configuración de la competencia lingüística y en los posteriores aprendizajes curriculares y sociales que tienen lugar a lo largo de la vida. Diversos informes, entre ellos el PISA, se han centrado en las peculiares circunstancias de la lectura en los adolescentes. Han llegado a la conclusión de que en España ésta es deficitaria respecto a otros países de la Unión Europea. Ahondando en estos datos, encontramos además que la mayoría de estudiantes españoles lee de forma obligada. Esto hace que su interés por la lectura sea nulo ya que no les resulta atractiva y no va más allá de un mero instrumento curricular de obligatoria ejecución en la escuela.

Siguiendo a Yubero y Larrañaga (2OIO) comprobamos cómo en muchos casos los alumnos españoles son capaces de descifrar el lenguaje escrito, pero ni leen de forma habitual, ni la lectura forma parte de su tiempo de ocio, ni son capaces de extraer los be- 
neficios que la lectura les ofrece. Esto se traduce en datos, cuanto menos, preocupantes. Así un tercio de los escolares que termina la Educación Primaria no sabe leer ni escribir correctamente, ni demuestra una adecuada capacidad para la comprensión del lenguaje escrito. Paradójicamente, como nos dice Chartier (2OIO) avanzamos hacia el sueño de la biblioteca universal mediante la acción de la revolución tecnológica.

A nuestro entender esto se debe a que vivimos en una sociedad que no presta demasiada atención a la lectura y en la que el éxito se basa en la economía, el reconocimiento personal, el consumo y el hedonismo. Los medios de comunicación nos están ofreciendo continuamente modelos de personajes de este tipo. Además, muestran abiertamente su desinterés por la lectura y se adscriben hacia otros ocios que les resultan más provechosos y a los que hay que dedicar un menor esfuerzo personal.

En consecuencia con lo que venimos señalando, cuando una persona se libera de las tareas obligatorias, lo que tiene que hacer para divertirse es salir, viajar, estar con los amigos, ver la televisión, conectarse a internet, etc. Entre estas actividades para el tiempo libre no encontramos, o solamente de una forma marginal, a la lectura. Ésta suele quedar relegada a los espacios de trabajo y de formación curricular, como una exigencia a cumplir en un tiempo absolutamente distinto a los términos en los que se define el ocio.

Es algo evidente que el lector se hace leyendo, y no se puede leer si no dedicamos parte de nuestro tiempo de ocio a la lectura. El hecho de valorar la lectura, pero no el tiempo de leer, puede ser una de las causas de que encontremos personas que, valorando la lectura, no lean voluntariamente. Son personas que no leen más que lo estrictamente necesario para completar su currículo formativo. También hemos de tener en cuenta las aportaciones de Gemma Lluch (2OIO) quien acertadamente denota que el joven del siglo XXI es objeto de una serie de cambios culturales que afectan a su crecimiento y por ende a su modo de entender el mundo y lo que éste contiene. Obviamente, la lectura está inserta en esta comprensión. Debemos pues combinar las nuevas tendencias que nos aporta Eloy Martos (2006), como prácticas lectoras basadas en fenómenos mediáticos o culturales con las lecturas tradicionales, las canónicas y con aquellas que son libremente escogidas por la persona lectora. Lo realmente importante es la promoción de la lectura, la combinación de alternativas será siempre positiva para esta labor promotora.

\section{El amor como tópico en la historia literaria}

A lo largo de la historia se ha tratado el tema del amor de manera distinta; entiéndase, se han producido distintas visiones histórico-culturales del amor hacia el otro o el mismo género. En la Antigüedad y hasta el Renacimiento, atravesando la Edad Media, el amor fue el concepto de un ideal; virtud y belleza eran el espejo de la mujer, una mujer idealizada. El amante estaba dispuesto a morir por su sentimiento. Ejemplos de este tipo de amor lo tenemos en las historias de Júpiter e Ío, Orfeo y Eurídice o Píramo y Tisbe en la época grecolatina. También lo encontramos en obras medievales como Cárcel de amor 
de Diego de San Pedro o en La Celestina de Fernando de Rojas. Ejemplos renacentistas serían la Beatriz de Dante o la Laura de Petrarca. En España merece especial mención Garcilaso de la Vega, quien en su soneto XXV, y en general en todos sus sonetos vinculados con la muerte de Isabel Freyre/ Elisa, expresa su deseo de morir después de la muerte de su amada para encontrarse con ella en el más allá.

Así pues en los Siglos de Oro, el amor se consideraba una enfermedad que conducía a la muerte; la pasión amorosa se interpretó como la primera enfermedad de la civilización europea: la melancolía. Además, el amor se entendía como algo pasional, breve e irracional y a ninguna persona se le hubiese ocurrido relacionarlo con el matrimonio. Éste era lo contrario, la decisión racional que se subordinaba a los intereses económicos; incluso entre las clases medias y altas el orden doméstico se subordinaba a la unión matrimonial.

En el siglo XVIII, por su parte, el amor ya no será sólo una cuestión de las clases más favorecidas, sino una cualidad interior para la que están capacitados todos los hombres. Es en esta época cuando comienza a fraguarse un modelo que sigue vigente hasta hoy; donde todo está permitido: las conquistas en cadena, el amor platónico, el amor furtivo, el destructor, y cuantas maneras se le ocurran al amante.

A juicio de Amparo Medina-Bocos (I991:26) “quizá sea el Romanticismo una de las épocas más decisivas para configurar el actual concepto del amor. En la literatura de esta época se ama al otro como ser total, incluyendo sus defectos. No se ama por la belleza, la situación económica o la disponibilidad, se ama por cómo es la persona ama-da, no por la consideración social que tenga".

En su trayectoria vital el hombre romántico frecuentemente se ve amenazado por la muerte que forma parte del destino trágico y determinado. El sentimiento del amargo desengaño se observa, por ejemplo, en el Canto a Teresa de José de Espronceda. En Don Álvaro o la fuerza del sino del Duque de Rivas, Leonor y don Álvaro son la pareja protagonista, impulsada por el amor puro, pero destinada a fracasar. Por su parte, Don Juan Tenorio de José Zorrilla es la versión donjuanesca más popular en la literatura española. De la condena de Tirso de Molina en el siglo XVII llegamos en el siglo XIX a la salvación por el amor puro.

En la época actual encontramos, igualmente, a numerosísimos autores que han tratado el tema del amor. Algunos autores reconocidos son Rosa Regás, Clara Sánchez, Arturo Pérez-Reverte, Mario Vargas Llosa, Rosa Montero, Antonio Muñoz Molina, Álvaro Mutis o Félix de Azúa. Otros con menor calidad literaria, pero con un éxito mundial de ventas, son Federico Moccia, Stephanie Meyer, Dan Brown, Patrick Rothffus, Javier Sierra o John Grisham.

Así pues, la historia de la literatura está llena de obras imprescindibles para entender el recorrido del amor: Tristán e Isolda de procedencia celta, Romeo y Julieta de Shakespeare, Las amistades peligrosas de Choderlos de Laclos, Orgullo y prejuicio de Jane Austen, Rojo y Negro de Sthendal, Madame Bovary de Flaubert, Ana Karenina de Tolstoi, Effi Briest de Theodor Fontane, La Regenta de Clarín, Pepita Jiménez de Juan Valera o Lolita de Nabokov, entre otras no menos importantes. 


\section{Tres referentes para el tratamiento del amor}

Dada la naturaleza descriptiva y comparativa de este trabajo, hemos escogido a tres autores muy diferentes en su manera de abordar el tema del amor. Puesto que estamos ante un constructo atemporal, hemos hecho un tratamiento del mismo a través de tres épocas diferentes (Antigüedad, Renacimiento y época actual).

Como primer referente sobre el tratamiento del amor en la literatura hemos elegido a Ovidio, quien en su mito de Píramo y Tisbe, nos ofrece la historia de los dos enamorados de una manera condensada, poética y llena de un gran lirismo. La historia puede llegar a hacer estremecerse al lector sensible.

Shakespeare, por su parte, va a versionar con Romeo y Julieta la historia de los amantes, mostrando con ella el patetismo y el afán de aparentar que se guardaba en los diferentes estamentos sociales de la época renacentista. Quizá sea esta historia del siglo XVI en la que el amor tiene un mayor componente lúdico. Éste alcanza gran intensidad al final de la obra pero, en los instantes iniciales, Romeo parece que se lo toma como algo meramente sexual y mudable.

Moccia, en nuestro tiempo, hace una adaptación de la historia añadiendo algunos componentes de naturaleza un tanto degradada que forman parte de la realidad amorosa actual. Es en esta historia titulada $A$ tres metros sobre el cielo, en la única en la que se nos ofrecen otras acciones secundarias.

Nuestro objetivo fundamental es que el alumnado se interese por las historias clásicas, una vez se ha demostrado que lee por gusto historias como las de Federico Moccia. Vamos a tratar de suscitar este interés fundamentalmente a través de los motivos temáticos por los que están compuestas las historias, dado que son muy similares.

El primer elemento que tienen en común serían los obstáculos que se les presentan a los enamorados para encontrarse. En ninguna de las tres historias las familias están de acuerdo con que los jóvenes se vean. Así en Píramo y Tisbe se dice: "La vecindad les hizo conocerse y dar los primeros pasos; con el tiempo creció el amor, y se habrían unido en legítimo matrimonio, pero se opusieron sus padres" (Ovidio. 1995: I45). En Romeo y Julieta, el enfrentamiento entre las familias se hace patente en el mismo comienzo de la obra: "En Verona, escena de la acción, dos familias de rango y calidad renuevan viejos odios con pasión y manchan con su sangre la ciudad"(Shakespeare. 20IO: 37). En A tres metros sobre el cielo, es la madre de Babi la que no quiere que su hija salga con Step. Lo demuestra el siguiente comentario que hace Raffaella sobre Step: “[...] Hace carreras de motos, firma justificantes falsos...Y además, ¿no has visto el morado que lleva debajo del ojo? Para mí que hasta le pega. No quiero que la vea más” (Moccia. 2008: 305).

Otro elemento que aparece en las tres historias es el deseo de lucha por parte de los enamorados. Ninguno de ellos está dispuesto a renunciar al amor. Vemos ejemplos de las tres obras:

Píramo y Tisbe: “¿Por qué te interpones entre dos enamorados, pared envidiosa? ¿Qué te costaba permitirnos unir por entero nuestros cuerpos o, si eso es demasiado, abrirte por lo menos para besarnos?" (Ovidio. 1995:I45). 
Romeo y Julieta: "Romeo: me oculta el manto de la noche y, si no me quieres, que me encuentren: mejor que mi vida acabe por su odio que ver cómo se arrastra sin tu amor"(Shakespeare. 2OIO: 72).

A tres metros sobre el cielo: "Step: hagamos locuras. Veámonos a las ocho en tu colegio. Espero despertarme a tiempo" (Moccia. 2008: 266).

Es también común la naturaleza del amor que se da en las tres obras. En todas ellas se nos presenta un amor incorruptible y virginal. Ejemplos de esto los tenemos en las tres lecturas. De este modo en Ovidio se dice: "Juntos dieron sus primeros pasos" (Ovidio. 1995: I45). En la obra de Shakespeare se habla de la juventud de Julieta y de que no conoce el amor: "Capuleto: mi hija nada sabe de la vida; aún no ha llegado a los catorce" (Shakespeare. 20I0: 48). En la novela de Moccia la referencia a la pureza de Babi es más explícita: "Babi: Si quieres decir que soy virgen, la respuesta es sí. ¡Y ahora llévame al colegio!" (Moccia. 2008: 275).

Finalmente, también coinciden los finales trágicos con reconciliación familiar posterior que se dan en las tres. En Píramo y Tisbe los amantes van a morir, igual que en Romeo y Julieta. En ambas, las familias se van a dar cuenta de su error demasiado tarde. En $A$ tres metros sobre el cielo, van a separarse de manera indefinida y el padre de Babi, Claudio, va a tomar un especial cariño por Step. Veamos los diferentes pasajes de sus finales.

Píramo y Tisbe: "Tisbe: Tu propia mano y el amor te han perdido, desgraciado. Tengo yo también una mano fuerte para esto solo, también tengo amor: éste me dará fuerzas para herirme" (Ovidio. 1995: I47).

Romeo y Julieta: "Julieta, al ver a Romeo muerto: Puñal afortunado, voy a envainarte. Oxídate en mí y deja que muera" (Shakespeare. 2010: 150).

A tres metros sobre el cielo: "Step: es hora de regresar a casa [...] con una única pregunta: ¿Volveré a estar alguna vez allí arriba, en ese lugar tan difícil de alcanzar? ¿Allí dónde todo parece más hermoso? Y en ese mismo instante, desgraciadamente, ya conoce la respuesta" (Moccia. 2008: 390).

A través de todos estos elementos comunes se pueden relacionar las tres obras. No obstante, esto no nos debe hacer olvidar que presentan niveles muy diferentes en cuanto a estilo narrativo, lenguaje utilizado y valor literario. La finalidad es que los alumnos pasen del lenguaje poco elaborado de Moccia al más bello de Shakespeare y, finalmente, den el salto a un estilo más compuesto y refinado como el que nos presenta Ovidio. Sinceramente, creemos que la mejor manera de conseguir esto es a través de un trabajo individualizado con cada alumno/a. Por este motivo, consideramos que, en este ámbito, la labor de los profesores de las diferentes especialidades es fundamental. Deben tratar de presentar todas las lecturas que propongan a sus alumnos de una manera atractiva.

A continuación, ofrecemos un breve análisis literario de cada una de las obras. Antes de tratar el argumento y los temas que se tratan en ellas, haremos una breve síntesis de la biografía de sus respectivos autores. 


\section{3.r.- El mito de Píramo y Tisbe}

Se trata de una obra escrita por Ovidio. Éste nació el veinte de marzo del año 43 a. C. en Sulmona, ciudad peligna de los Abruzzos, al este de Roma. El año de su nacimiento coincidió con la muerte de Cicerón y la formación del segundo triunvirato.

Su formación retórica le forjó en el gusto por la selección de las palabras y por la disposición artística y rítmica de las mismas. Con todo Quintiliano creyó que Ovidio habría sido mejor poeta si hubiera controlado su talento y no al revés. Sobre al año 25 a.C., es decir a los dieciocho años ya andaba recitando su primera versión de los Amores y ahí empezó a crecer su popularidad de poeta que llegó como nadie al corazón de la sociedad urbana de la Roma de entonces.

A la obra de madurez del poeta corresponden Las metamorfosis, extenso poema en hexámetros que recoge diversas historias y leyendas mitológicas sobre el tema de las transformaciones. Representan el primer intento suyo por conseguir la excelencia en la poesía erótica y en el verso elegíaco. El poema es un compendio de leyendas clásicas y constituye una verdadera fuente de mitología grecolatina.

Los mitos ovidianos tuvieron un gran encanto para todos los escritores que los tratan. Las fábulas de Hero y Leandro, Jasón y Medea, Píramo y Tisbe, Venus y Marte, Faetón, Narciso, Orfeo y muchas otras lograron tanto favor que han sido reescritas en numerosas versiones.

Quizás el más popular de los mitos ovidianos haya sido el de los amantes babilonios: Píramo y Tisbe. Su asunto es profundamente romántico y se encuentra condensado en unos cuantos versos. El estilo con el que está escrito estimula la imaginación de cuantos lo leen por constituir una de las fábulas más sencillas y más humanas de todas las contenidas en Las metamorfosis. Tuvo tanta popularidad en su época que sus protagonistas se convirtieron muy pronto en símbolos del amor desdichado. Además, de esta historia va a surgir el mito del color de las moras. Las mismas serán negras -rojo sangre unido al púrpura original- cuando ya sean comestibles, como recuerdo de la sangre vertida por los dos amantes. La atracción que suscitó el texto dio origen a múltiples imitaciones, muchas de ellas acompañadas de una interpretación alegórica; otras adolecen de abundantes pormenores superfluos. Todas, sin embargo, intentan duplicar a su manera y para sus propósitos los elementos trágicos y muy humanos del original.

\section{2.- Romeo y Julieta}

Composición teatral escrita por el dramaturgo inglés William Shakespeare. Éste nació el 23 de abril de I564 en Stratford-upon-Avon. Está considerado como uno de los más grandes escritores de todos los tiempos. Hijo de un comerciante de lanas, se casó muy joven con una mujer mayor que él, Anne Hathaway.

Refiriéndonos a una de sus obras cumbre, Romeo y Julieta, hemos de señalar que ésta se ha contado de diversas maneras y siempre parece haber sido atrayente, acaso por- 
que pertenece a la memoria colectiva y trasciende las distintas versiones de que ha sido objeto. Comoquiera que sea, la trágica leyenda tiene antecedentes en la mitología griega (Hero y Leandro, Píramo y Tisbe) y en algunas leyendas medievales, aunque es en Las efesíacas de Jenofonte de Éfeso (siglo II) donde se aprecia un esquema argumental que podría haber dado origen a la historia de los amantes de Verona.

Uno de los rasgos más sobresalientes que diferencia a Romeo y Julieta de sus predecesoras es su exuberancia lingüística. En ella hay una gran adecuación entre lengua y personaje y también una generosa exhibición de fuerza poética y retórica. Esto se demuestra en que la obra comienza con un soneto y es también en un soneto, seguido de un cuarteto, como se hablan por primera vez los dos amantes.

Pero no es solo el lirismo lo que destaca en la obra de Shakespeare, ya que éste es el autor que más se centra en lo privado de las relaciones amorosas. Primero, la familia Montesco y, especialmente, Romeo. Los padres parecen intrigados por saber qué le pasa a su hijo: diversas referencias hacen suponer que es un joven sociable, cortés y estimado, pero últimamente está cambiado y se muestra retraído.

A través de su conversación con Benvolio observamos no sólo que está enamorado, sino que es rechazado por la cruel amada (Rosalina). La estilización del diálogo, con sus clichés y sus tópicos, confirma que el estado de Romeo viene a ser una parodia del amor petrarquista tan cultivado por la poesía de tiempos de Shakespeare. Se puede añadir que Romeo es un enamorado del amor y, en definitiva, un enamorado de sí mismo. Pero, a su vez, esa presentación privada remite a unos usos públicos: Romeo sigue una moda y lo que adopta es una pose social y una actitud personal.

La presentación de Julieta se realiza en dos etapas sucesivas. Primero, indirectamente, en el diálogo entre su padre y el conde Paris, su pretendiente, y después, directamente, en la escena tercera del acto primero. Julieta aún no ha cumplido los catorce años, y su padre prefiere aplazar la boda dos años pero su intervención es mínima y su presencia se ve dominada por la de su madre y, especialmente, la del ama. Sin embargo, es de Julieta, de su pasado, presente y futuro, de lo que se habla en esta escena. El ama utiliza un vocabulario pobre, con una sintaxis ilógica y hace muchas digresiones en sus parlamentos. Estas características dan la impresión de que es una persona simple e iletrada, aunque su tono denota afecto y humanidad.

Más adelante en la obra, con el posterior enamoramiento de Romeo y Julieta se aclara la importancia de lo social en la misma. El primer beso de ambos en la fiesta los abstrae de su mundo circundante y, cuando más tarde corra al balcón de Julieta, Romeo tendrá que esconderse de sus mejores amigos (que se referirán a él y a su amor por Rosalina en términos abiertamente sexuales). Una vez concertada la boda con Julieta, Romeo se reúne con Benvolio y Mercucio, pero no les revela su secreto. Mercucio morirá sin conocerlo y Benvolio desaparecerá de la obra tras explicar públicamente la muerte de Mercucio y de Tebaldo.

La naturaleza del amor entre ambos exige el secreto, pero, a su vez, éste aísla a la pareja. Mercucio muere creyendo que Romeo sigue amando a Rosalina pero, en cual- 
quier caso, su cinismo amoroso no le habría permitido comprender a los protagonistas. El ama ayuda a Julieta, pero su concepto puramente sexual del amor nos da a entender que lo hace a modo de una celestina, como no deja de observar Mercucio. El fraile accede a casar a Romeo y Julieta, pero no porque sea favorable a su amor por sí mismo, sino por una razón práctica: la reconciliación de las familias a que podría llevar su matrimonio. En cuanto a Capuleto y su mujer, la violencia con que tratan a Julieta porque ella se resiste a casarse con Paris deja a su hija en manos del ama, quien a su vez la abandona al proponerle que se case con Paris. Por su parte, el fraile también abandonará a Julieta en su tumba junto al cadáver de Romeo.

Sin embargo, a Shakespeare no parece interesarle la condena moral de estos personajes por sí misma (de hecho, ninguno de ellos obra con maldad), sino más bien por su efecto dramático: el patetismo suscitado por el total aislamiento que unos y otros dejan a los protagonistas hasta el final de la obra; un final en el que los presentes lamentan el infortunio y celebran la reconciliación, pero no responden a un amor cuya excepcionalidad les supera.

\section{3.-A tres metros sobre el cielo}

Nos encontramos ante una de las novelas más vendidas del panorama literario actual. Su autor es Federico Moccia. Éste nació en Roma, Italia, el ro de noviembre de I963. Empezó trabajando en el mundo artístico como escenógrafo y guionista de programas de televisión, pero poco a poco se fue adentrando en el mundo de la literatura. La obra que aquí nos ocupa, $A$ tres metros sobre el cielo, publicada en I992, está considerada en la actualidad un texto de referencia para el público juvenil puesto que representa la dimensión ideal del amor libre y sin barreras. Entre las calles de la ciudad eterna son muchos los seguidores que recorren la llamada "ruta Moccia", rememorando los lugares en los que se desarrolla la trama de sus novelas.

La novela comienza con Babi. Nuestra protagonista es una joven que se prepara para ir a la escuela con su padre y con su hermana pequeña. Por otro lado, tenemos a Step que, como otro día cualquiera, coge la moto y se dispone a dar una vuelta. Cuando ella va montada en el coche mirando por la ventanilla, él no puede evitarlo y le habla. Todo lo que se dicen no parece tener importancia; sólo nos centramos en la intensidad de sus miradas.

Babi es una chica rubia, de ojos azules, con una familia estable y adinerada. Tiene un carácter dulce y es una chica tranquila. Step es lo contrario: un chico problemático aficionado a las motos y a todos los peligros. Sin embargo, en el fondo tiene un gran corazón. El motivo por el que su comportamiento es violento proviene de su pasado.

Tras unos cuantos encuentros entre ellos, se enciende la llama del amor. Un amor intenso, peligroso y apasionado, y sobre todo difícil de llevar. A pesar de todo, se trata de una relación que parece condenada al fracaso dada la diferencia de clases y de comportamiento. Se trata de la historia del primer amor entre dos adolescentes de la época actual. Moccia trata de envolvernos en el mundo sentimental de la juventud de hoy en día. 
En el libro hay constantes referencias a marcas de ropas y de complementos y a diferentes lugares muy valorados por los jóvenes. Además, se utiliza un vocabulario sencillo y con algunas palabras coloquiales y, en ocasiones malsonantes, en consonancia con las que utiliza el público al que va dirigida. Va a ser un elemento muy propio del mundo contemporáneo, los grafitis, los que van a abrir y a cerrar el círculo de la historia amorosa entre Babi y Step. Así la novela comienza con: "Cathia tiene el mejor culo de Europa. El grafito rojo brilla con toda su desfachatez sobre una columna del puente de la avenida de Francia" (Moccia. 2008: 7), y va a llegar al final con otro que dice: "Arriba, en la blanca columna del puente, una inscripción resalta sobre todas las demás, imborrable: "Tú y yo... a tres metros sobre el cielo"” (Moccia. 2008: 36o).

Además de la historia principal, en la obra encontramos otras secundarias. De entre ellas destaca la que tiene lugar entre Pollo y Pallina. El primero es el mejor amigo de Step y la segunda lo es de Babi. Van a entablar una relación amorosa que se verá truncada por la muerte de Pollo casi al final de la obra. Esta muerte hará que Babi y Pallina se separen ya que a la segunda se le agriará el carácter con la muerte de su novio. La separación entre Babi y Pallina es parecida a la que se producirá entre Babi y Step cuando la chica conozca a Alfredo, un novio que se corresponde más con su clase social. Nuestro autor deja abierta la historia, de manera que le permita introducir la segunda parte de la misma: Tengo ganas de ti.

\section{Investigación realizada}

\section{I.- Objetivos}

Objetivo general:

Comprobar y analizar la actitud y conocimiento del alumnado de Educación Secundaria Obligatoria hacia el tema del amor como tópico literario.

Objetivos específicos:

Primero. Conocer las preferencias lectoras del alumnado de cuarto de la E.S.O. La consulta se hará sin ningún tipo de condicionamiento previo. Se pretende comprobar de una manera lo más fidedigna posible cuál es la realidad lectora de los jóvenes actuales de entre quince y dieciséis años.

Segundo. Averiguar si el alumnado de cuarto de la E.S.O. es capaz de relacionar textos de diferentes épocas literarias. Este objetivo persigue descubrir cuál es el nivel lector de cada alumno.

Tercero. Analizar si el alumnado de cuarto de la E.S.O. posee capacidad de reflexión con respecto a los temas literarios. El objetivo es tratar de promover en el alumnado una conciencia crítica sobre los distintos temas literarios y la manera en que han sido abordados en cada época. 


\section{2.- Metodología}

El peso de la tradición, la ausencia de pautas consolidadas para la innovación en el tratamiento didáctico del hecho literario, la permanencia de una fijación historicista en el diseño curricular y la inercia metodológica que abruma al profesorado son las causas que determinan la presencia continuada de un desafortunado planteamiento didáctico basado en la memorización de datos que resulta ineficaz para lograr la implicación del lector en la recepción y hacer de su formación la clave para que sepa y pueda actuar con la autonomía que le confiera una desarrollada competencia lectora. Se trata, pues, de conseguir un giro en la concepción de la literatura, reubicando los contenidos específicos de su formación.

Nosotros hemos optado siempre por trabajar con los textos y no utilizar datos estereotipados. Nuestra metodología se ha basado en utilizar el intertexto lector de los alumnos. Éste sirve para potenciar las habilidades de recepción de los textos y de valoración personal. Lo interesante es que de un texto pasen a otro y que a través de su interrelación, consigan formarse una opinión crítica sobre cada uno de ellos. Esto sólo se consigue a través de la lectura de los textos, sin demasiadas explicaciones teóricas. Esta técnica constituye, a nuestro parecer, una metodología muy sugerente de aproximación didáctica a la formación lectora.

\section{4•3-- Población y muestra}

La población de estudio definida en este trabajo comprende al alumnado de Educación Secundaria del IES Amparo Sanz, situado en la ciudad de Albacete (Castilla-la Mancha). Éste es un centro que cuenta con unos 800 alumnos/as. Desde el punto de vista de los procesos de la enseñanza y el aprendizaje, la población escolar de este instituto es un colectivo que, en términos generales, presenta pocas dificultades añadidas sobre las que son propias de cada una de las etapas de la ESO y bachillerato. Las dificultades que últimamente se están planteando, fundamentalmente a nivel de enseñanza, derivan de la incorporación de un número más elevado de alumnos de otras etnias.

La muestra escogida para llevar a cabo el trabajo corresponde con estudiantes de cuarto curso de Educación Secundaria Obligatoria. La misma está compuesta por $5^{8}$ sujetos, siendo 26 de éstos mujeres y $3^{2}$ varones. Se trata, por lo general, de alumnos/as altamente motivados en casi todas las asignaturas. Concretamente, en lengua española y literatura se muestran participativos, creativos y normalmente interesados en lo que se les explica. Además, según la tutora de los alumnos, cerca del $25 \%$ de ellos suelen leer habitualmente (sobre todo literatura juvenil y actual). Es este grupo lector el que presenta una mayor capacidad artística y creativa y el que motiva al resto. 


\section{4.- Instrumentos de medida}

\section{Encuesta de preferencias lectoras}

Con esta encuesta el objetivo era conocer cuáles eran los libros y los autores más conocidos por el alumnado de cuarto de la ESO. El instrumento utilizado es bien sencillo, una tabla con diez filas y dos columnas. En la primera columna tenían que poner el título del libro y, en la segunda, el autor del mismo. Se les dejaron diez huecos porque estimamos que serían suficientes. En muchos casos lo fueron, pero hubo otros alumnos que siguieron rellenando fuera de la tabla.

\section{Cuestionario sobre las lecturas}

El siguiente instrumento de medida que utilizamos fue un cuestionario sobre las lecturas. El título del mismo es "cuestionario sobre modelos clásicos y modernos en el tratamiento del amor". Está compuesto por dos partes. En la primera, se pide a los/as alumnos/as que, a través de la lectura de una serie de fragmentos que se les proporcionan, identifiquen el título de la obra al que pertenecen. Además, al final se les pide que señalen si les gusta este tipo de literatura. Por otro lado, en la segunda parte se les solicita que identifiquen el libro al que se vinculan una serie de parejas enamoradas y se les pregunta si lo han leído. Con cada pareja, se les va requiriendo el tema principal del libro y si hay otros temas secundarios. Finalmente, se les pregunta si es de su gusto esta literatura. Si responden que sí tienen que señalar el porqué. Las respuestas afirmativas se acotan, por motivos estadísticos, a una serie de causas (calidad literaria, tema del amor, lecturas atemporales y facilidad lectora).

\section{Debate sobre las lecturas}

El último instrumento que se utilizó fue un debate sobre la lectura de la novela de Moccia que habían tenido que leer anteriormente. Se organizó en dos partes. En la primera lo que hicimos fue pedir opinión sobre la obra del autor italiano. Las respuestas fueron bastante dispares, aunque aproximadamente la mitad de los presentes respondieron que la lectura había sido de su agrado. Sin hacer ninguna valoración sobre sus respuestas, procedimos a leerles la historia de Píramo y Tisbe. Los/as alumnos/as la escucharon con gran atención y, como nos dijeron después, fue muy de su agrado. Comenzamos a preguntarles sobre el tema del mismo. La respuesta generalizada fue sobre el amor imposible. Les pedimos que comparasen el lenguaje que se utilizaba en una y otra obra. Debatimos sobre el panorama actual de la novela española, sobre el poder que tienen las editoriales, su calidad literaria y el gusto de los lectores. Extrajeron conclusiones bastante sensatas e hicieron varias críticas sobre la realidad del mercado literario actual.

En la segunda parte del debate, un profesor invitado del departamento de inglés, explicó de una forma bastante resumida la historia de Romeo y Julieta. Seguidamente, les preguntamos sobre Shakespeare para que nos dijeran qué obras conocían de él. La mayoría de los/as alumnos/as conocían pocas obras y casi ninguno había leído nada. A 
través del tema del amor pusimos en relación la obra de Moccia y la del dramaturgo inglés. Les explicamos que la novela actual no es más que una versión poco cuidada de un tópico atemporal como es el del amor. Empezamos a señalarles aspectos del texto de $A$ tres metros sobre el cielo que no eran más que una modernización degradada de Romeo y Julieta. Para ello, utilizamos pasajes de las dos obras. Seleccionamos parejas de alumnos e hicimos que cada uno leyese un fragmento del mismo momento de la historia de cada autor. Les preguntamos cuál les gustaba más. Casi todos señalaron que los de Shakespeare ya que el lenguaje estaba más cuidado y se expresaban los sentimientos de forma más intensa. Muchos/as alumnos/as quedaron bastante satisfechos con el diálogo mantenido y nos aseguraron que, en cuanto tuvieran tiempo, iban a leer Romeo y Julieta. Otros, sin embargo, nos hicieron saber que les apetecía leer más la obra de Moccia Tengo ganas de ti, continuación de $A$ tres metros sobre el cielo.

\section{5.- Procedimiento}

El procedimiento que hemos utilizado en esta investigación es mixto. Según Miles y Huberman (I994) es un tipo de método que combina el análisis deductivo e inductivo. El proceso analítico parte de un esquema general previo (en este caso comparativo), cuyo contenido va especificándose en la medida que la persona investigadora interacciona con el texto y su contexto.

Procedemos a describir cada una de las fases del trabajo de investigación:

Fase preliminar: elección de una metodología literaria comparativa para promover la lectura.

Para ello, nada mejor que enfocar nuestro estudio en los lectores. Una vez que supiéramos lo que los/as alumnos/as leen por placer, podríamos conducirlos hacia lecturas más instructivas. Partimos de "sus" textos para llevarlos a "nuestros” textos.

\section{Fase primera: confección y utilización del instrumento de recogida de información.}

El primer instrumento de recogida de información utilizado fue una encuesta de preferencias lectoras. Se les proporcionó a los/as alumnos/as una tabla con diez filas en blanco para que la rellenaran con los libros -y sus correspondiente autores- que más les hayan hecho disfrutar a lo largo de sus vidas. Muchos alumnos no conocían el nombre del autor del libro que ponían y, debido a la necesidad de analizar los datos de forma exhaustiva, tuvo que ser señalado a posteriori. Esta encuesta se pasó en la primera sesión que tuvimos con ellos.

Fase segunda: cuestionario sobre modelos clásicos y modernos en el tratamiento del amor.

Se confeccionó un cuestionario con preguntas sobre diferentes parejas literarias que han sido consideradas símbolos del amor. No se avisó previamente de la realización del mismo al alumnado, ni tampoco del objetivo que perseguíamos, puesto que preten- 
díamos descubrir los conocimientos reales que tenían. Del mismo modo, nuestra intención era conocer la opinión de los encuestados expresada espontáneamente, y desprovista de todo condicionamiento externo. Este cuestionario tuvo lugar durante tres sesiones diferentes debido a que eran demasiados alumnos para hacerlo todos al mismo tiempo.

\section{Fase tercera: lectura de A tres metros sobre el cielo de Federico Moccia}

En otra sesión, y consensuado con la profesora de los alumnos/as, se les mandó que leyeran la novela del autor italiano. Se perseguía fundamentalmente que los chicos de la clase leyesen la historia (la mayoría de las chicas ya lo habían hecho). Para asegurarse de lo que lo hicieran, se les encomendó un trabajo que tendría hasta dos puntos en la calificación final. Hemos de ser conscientes de que el alumno trabaja mejor motivado con las calificaciones.

\section{Fase cuarta: seguimiento de la entrega de los trabajos por parte del alumnado}

Al llegar el día de la recogida de trabajos tuvimos la suerte de que todos los estudiantes lo habían realizado. En el mismo se les pedía que dieran su opinión personal sobre el libro. Las opiniones fueron dispares. Parece que a los alumnos más trabajadores el libro les pareció vacío y falto de coherencia interna, sin embargo, los menos estudiosos señalaron que les había parecido un buen libro ya que se identificaban con los protagonistas. Tomamos los trabajos, ya corregidos, para preparar la siguiente fase.

\section{Fase quinta: debate con los alumnos sobre los libros de amor}

Algunos días después de la entrega del trabajo, tuvimos un debate con los/as alumnos/as que habían colaborado con nosotros. Como eran $5^{8}$, la profesora pidió reunirse con ellos/as en el salón de actos. Acudieron la mayoría. Sólo faltaron 2 personas por diferentes motivos. En el debate intervinimos cuatro personas: dos profesores de lengua y nosotros. De los profesores de lengua, una era la titular de los/as alumnos/as y otro era un compañero del departamento de inglés que se ofreció para participar en el debate sobre Romeo y Julieta. Fue una sesión de unos sesenta y cinco minutos (se tomaron diez del recreo) y resultó muy productiva ya que, al final de la misma, eran numerosos los/as alumnos/as dispuestos a leer diferentes historias de amor y, sobre todo, la obra de Shakespeare.

\section{6.- Análisis y discusión de resultados}

Vamos a analizar, a continuación, cuáles fueron los resultados de los diferentes instrumentos de medida para valorar el interés de los alumnos/as por la literatura de amor.

Con respecto a la encuesta sobre preferencias lectoras, hemos de señalar que éstos son los diez autores que más éxito tuvieron entre esta muestra de adolescentes: Jordi Sierra i Fabra - 34 lecturas, Federico Moccia - 29 lecturas, Stephanie Meyer - 29 
lecturas, Laura Gallego - I9 lecturas, J. K. Rowling - I3 lecturas, Joan Manuel Gisbert - 8 lecturas, Tolkien - 7 lecturas, Carlos Ruiz Zafón - 7 lecturas, Jack London - 7 lecturas y John Boyne -6 lecturas.

Como se puede observar, destacan los autores actuales y comerciales como Federico Moccia, Stephanie Meyer, J. K. Rowling, Tolkien o Boyne. También aparecen otros autores que se dedican fundamentalmente a escribir literatura juvenil como son Jordi Sierra i Fabra, Laura Gallego, Joan Manuel Gisbert y Carlos Ruiz Zafón. Su presencia, parece evidente, se debe a que los profesores de educación secundaria suelen mandar leer sus obras de forma obligatoria. El autor que más destaca, a nuestro juicio, sería Jack London. Parece que, como Enid Blyton, ha conseguido que sus obras sean muy leídas durante varias generaciones.

Observando los datos de un modo comparativo entre chicos y chicas, nos percatamos fundamentalmente de que, mientras Federico Moccia es el más leído por las chicas, en los chicos no aparece siquiera entre los diez primeros. Éstos prefieren, por su parte, obras de literatura fantástica ya que Tolkien ocupa una posición privilegiada entre sus preferencias lectoras. En las chicas este autor aparece en una posición muy retrasada. Lo que es también observable es que las chicas tienen más afición a la lectura porque, cuando sumamos sus preferencias a las de los chicos, tenemos a Moccia situado en segundo lugar, mientras que Tolkien aparece el séptimo.

Por otro lado, la misma encuesta nos reveló los títulos más leídos por los adolescentes seleccionados. Veamos los diez primeros:

Lágrimas de sangre - 26 lecturas, A tres metros sobre el cielo - I3 lecturas, Harry Potter - I3 lecturas, Crepúsculo - II lecturas, Elniño con el pïama de rayas - 8 lecturas, Memorias de Idhún - 7 lecturas, Campos de fresas - 6 lecturas, Tengo ganas de ti- 6 lecturas, Perdona si te llamo amor - 6 lecturas, Luna nueva - 6 lecturas.

Respecto a las diferencias entre chicos y chicas, sucede lo mismo que anteriormente. En los chicos no encontramos títulos de amor (ni Crepúsculo, ni ningún título de Federico Moccia), mientras que en las chicas ocupan las primeras posiciones y muy destacados respecto a los demás. De la misma manera, un título como El señor de los anillos ocupa el tercer lugar en la encuesta de los chicos mientras que sólo una chica lo ha leído. Es evidente que hay diferencias entre sexos respecto a los gustos lectores.

El cuestionario sobre modelos clásicos y modernos en el tratamiento del amor, lo vamos a analizar de una manera comparativa entre chicos y chicas. Además, esta comparación se va a realizar en dos partes. La primera teniendo en cuenta el número de fragmentos acertados y la segunda el número de parejas que reconocen de forma atinada.

Analizando los datos obtenidos del reconocimiento de textos, observamos que no hay ningún alumno/a que reconozca las seis muestras ofrecidas aunque, también es cierto, tampoco hay nadie que no reconozca al menos una. Las chicas tienen unos índices más altos de acierto ya que, de las veintiséis, encontramos que tres reconocen cinco fragmentos, diecinueve cuatro, dos tres y las últimas dos, únicamente reconocen dos. Así pues, son una amplia mayoría -24 alumnas- las que reconocen tres o más fragmentos (en datos 
estadísticos estaríamos hablando de un 92,30\%) Por su parte, los chicos presentan unos resultados un poco peores ya que de los treinta y dos cuestionados, quince reconocen cuatro fragmentos, ocho tres, cinco dos y cuatro sólo uno. De este modo, son 23 alumnos los que identifican tres o más textos (en datos estadísticos estaríamos hablando de un 7т,87\%). Este es otro testimonio que demuestra que las alumnas están más interesadas, en general, por la lectura y, en particular, por las historias de amor.

En la segunda parte del cuestionario, basado en el reconocimiento de parejas amorosas, los resultados son similares aunque ofrecen mejores datos para los chicos. Asimismo, hemos de señalar que, en esta parte, encontramos algunos alumnos que reconocen todas las parejas y también otros que no reconocen ninguna. Las chicas siguen mostrando un rendimiento alto ya que, de las veintiséis, tenemos que once aciertan las siete parejas, cinco seis, cinco cinco, dos cuatro, una una y dos ninguna. En consecuencia, son $2 \mathrm{I}$ alumnas las que reconocen cinco o más parejas (esto supone un 8o,76\%). Por su parte, los chicos obtienen resultados ligeramente superiores. Así, catorce reconocen las siete parejas, cinco seis, ocho cinco, dos cuatro y tres tres. En resumen, 27 alumnos identifican cinco o más parejas (estamos hablando de un 84,37\% del total).

A pesar de los buenos resultados que arroja este cuestionario, nos gustaría destacar que, en la segunda parte del mismo, había que informar también sobre si habían leído la obra que protagonizaba la pareja. Pues bien, a esta pregunta respondieron solamente veintidós de los/as cincuenta y ocho alumnos/as que fueron cuestionados. Estaríamos hablando de que únicamente el 37,93\% de los/as alumnos/as respondieron a la pregunta. Esto nos muestra lo importante que es desarrollar la comprensión y la competencia lectora y las carencias que presentan nuestros estudiantes de educación secundaria en este aspecto.

El debate establecido con los/as alumnos/as fue, a nuestro juicio, la experiencia más enriquecedora y productiva de todos los instrumentos de medida empleados. A pesar de que el número de alumnos/as era muy elevado, podemos decir que todos salimos satisfechos del mismo. Cada alumno/a dio diferentes opiniones sobre sus temas literarios preferidos, sobre el mercado literario actual, sobre la necesidad de la obligatoriedad o no de la lectura, sobre los elementos que más les habían llamado la atención de la obra de Moccia y sobre las diferentes estrategias para promover la lectura.

\section{Conclusiones}

Tras analizar los datos obtenidos, y a modo de conclusiones generales, podemos extraer las siguientes:

- Se deben seguir haciendo más estudios de este tipo incidiendo en los gustos de los jóvenes a la hora de promover la lectura. En nuestro caso, hemos 
conseguido que los/as alumnos/as, partiendo de sus preferencias lectoras, se acercaran a textos más clásicos. Es muy importante no perder de vista que trabajamos para ellos/as.

- Unida a esta competencia lectora, debemos mejorar los niveles de comprensión lectora. Es importante que los/as alumnos/as entiendan lo que leen ya que, de esa forma, aumentarán sus niveles apreciativos, críticos y creadores. A este respecto, siguiendo a Manuel Vega (I990), apostamos por un modelo psicológico de la lectura que pasa por enseñar a comprender cualquier forma de lenguaje.

- Hay que intentar dar un cambio a la hora de explicar la literatura. La metodología, a nuestro juicio, debe centrarse más en trabajar con los textos y no desde fuera de ellos. Debemos evitar imponer juicios sobre determinadas lecturas y hemos de procurar que sean los estudiantes los que obtengan sus propias conclusiones.

- La lectura de los textos implica una técnica que hay que trabajar con el alumna-do. Es una destreza que implica una maduración personal. Su encanto no se extingue con los años, sino que aumenta y, si es verdad que pensamiento y lenguaje se desarrollan juntos, y en la medida en que ese desarrollo facilita la regulación de la conducta, la lectura puede devenir en una escuela de civismo, de tole-rancia, de participación, de compromiso con la naturaleza, con el patrimonio cultural y, fundamentalmente, con una existencia mejor, de manera que hagamos realidad las palabras de Flaubert: "Leed para vivir".

- La literatura comparada resulta un marco bastante efectivo para la formación literaria del alumnado. A este respecto, según señala Claudio Guillén (2005:39) "la investigación de las relaciones dialécticas entre diversas obras conduce al enriquecimiento progresivo de sus elementos constitutivos".

- Los tópicos literarios resultan una buena manera de promocionar la lectura. En este tipo de literatura se tratan temas universales de los que todas las personas tienen unas mínimas nociones. Por este motivo, pueden resultar más atrayentes que la literatura más especializada. En nuestro caso, hemos conseguido que los/as alumnos/as sean capaces de establecer relaciones entre textos sirviéndonos del tópico del amor.

- Resulta evidente que los/as alumnos/as conocen más los libros actuales que los clásicos. Ya que tenemos la seguridad de que muchas de estas lecturas son de su agrado, ¿por qué no se introducen en el currículo estas lecturas? 
La propuesta actual consiste en obligarles a leer autores que escriben para jóvenes y que muchos de ellos ni les agradan. ¿Por qué no permitirles que sean ellos quienes escojan su propia literatura? Entiéndase que no se trata de eliminar los libros clásicos del mundo escolar sino de integrarlos con otros más modernos que los atraigan hacia el mundo lector.

- Hemos de fomentar la igualdad de géneros a la hora de leer. Debemos evitar los clichés y los tópicos que sugieren que hay un tipo de literatura para cada género. De este modo, por ejemplo, podríamos introducir a los chicos en la literatura de amor y a las chicas en la literatura fantástica. En nuestra investigación, hemos podido observar cómo la visión sobre la literatura de amor que tenían los chicos al principio ha cambiado sustancialmente: ha pasado de no interesarles nada, a ser apreciada en gran manera.

Como se puede apreciar, toda esta serie de conclusiones se alinean con la idea acuñada por Mendoza Fillola (2OOI) de que no es suficiente con "enseñar literatura" sino que es necesario "enseñar a apreciar la literatura", en el sentido de disfrutar y valorar las obras literarias. Para ello, hemos tenido en cuenta la recepción literaria personal de cada alumno/a, valorando sus conocimientos y sus referencias culturales previas.

En este sentido, hemos conseguido que el alumnado se haya implicado emocionalmente con los textos y que se haya producido una proyección de sus sentimientos particulares en los sentimientos de los principales personajes de los textos. Es decir, como señala Mata Anaya (2004:I35) hemos logrado que "su mundo interior se les ofrezca más transparente y que las arduas relaciones con la realidad externa resulten más asequibles, más intensas, más satisfactorias".

Del mismo modo, a través de la lectura de los textos, hemos intentado que los/as alumnos/as se atrevan a mirar la realidad con más osadía, amplitud y sagacidad, así como a que descubran su identidad, con todos los deseos, pasiones y dudas que llevan dentro. Hemos tratado, en fin, de que los reductos de sus sensaciones broten espontáneamente y se desplieguen sin limitaciones o pudores.

Con todo lo dicho, y según señalan los profesores López Valero y Encabo Fernández (2002: 87), creemos que debemos potenciar en los/as alumnos/as la consecución de una buena competencia literaria. En este sentido "tenemos que intentar que la aproximación a los textos literarios sea progresiva y no forzada. [...]. El acercamiento a los aspectos literarios se debe de hacer desde un planteamiento funcional, en ningún caso memorístico u obligado".

En consecuencia, según señalan Reyzábal y Tenorio (I992), hemos de entender la lectura como un juego. La obra literaria se debe concebir como un acto sin consecuencias y su lectura una liberación de la tensión que implicaría participar en su trama. Toda palabra es un vínculo con los otros y por lo tanto puede liberarnos lúdicamente de la opresión y la rutina de los roles cotidianos. En palabras de Wellershoff (I976) "la literatura es un 
soñar despierto que posee la magia y la seriedad de una intuición de lo real-posible, y así completa la incompleta experiencia directa y personal”.

Por último, no debemos olvidar que para la consecución de estos objetivos, es necesario remarcar el trascendente papel a la hora de promover la lectura que debe tener el Estado, en todos sus órdenes de gobierno, y también otros sectores de la sociedad, incluidos empresarios, sindicatos y otras organizaciones institucionales. De entre todos estos miembros, debe destacarse por su importancia la figura del profesor/a que actúa como mediador entre los textos literarios y los alumnos/as. De la actitud que éste/a asuma al tender el puente entre los libros y éstos/as dependerá la respuesta de éstos/as y la manera en que adopten la lectura como un elemento indispensable en sus vidas. 


\section{Referencias bibliográficas}

- Chartier, R. (20іо): “Aprender a leer, leer para aprender”. Nuevo mundo, mundos nuevos, Io. Consultado el 28/12/2OIO en http://nuevomundo.revues.org/5862I

- Guillén, C. (2005): Entre lo uno y lo diverso, Barcelona, Ed. Tusquets.

- Iser, W. (I987): Elproceso de lectura, Madrid, Ed. Taurus.

- Jauss, H.R. (1977): Experiencia estética y hermenéutica literaria, Madrid, Ed. Taurus.

- López Valero, A. y Encabo Fernández, E. (2002): Introducción a la didáctica de la lengua y la literatura, Barcelona, Ed. Octaedro.

- Martos, E. (2006): “Tunear los libros: series, fanfiction, blogs y otras prácticas emergentes de lectura", Ocnos, 2, pp 63-77.

- Medina-Bocos Montarelo, A. (I99I): Temas constantes en la literatura española, Madrid, Ed. Akal.

- Lluch, G. (2010): “Un nuevo lector, una nueva lectura, unos nuevos formatos”. En G. Lluch (Ed.). Las lecturas de los jóvenes. Un nuevo lector para un nuevo siglo, Barcelona, Anthropos, pp. 7-38.

- Mata Anaya, J. (2004): El rastro de la voz y otras celebraciones de la lectura, Granada, Ed. Universidad de Granada.

- Mendoza Fillola, A. (2OOI): El intertexto lector. El espacio de las aportaciones del texto con las del lector, Cuenca, Ed. Universidad de Castilla-La Mancha.

- Moccia, F. (2008): A tres metros sobre el cielo, Barcelona, Ed. Planeta.

- Ovidio (r995): Las metamorfosis, Madrid, Ed. Alianza.

- Reyzábal, M.V. y Tenorio, P. (1992): El aprendizaje significativo de la literatura, Madrid, Ed. La Muralla.

- Shakespeare, W. (2OIO): Romeo y Julieta, Madrid, Ed. Austral. 
- Vega, M. (I990): Lectura y comprensión. Una perspectiva cognitiva, Madrid, Ed. Alianza.

- Wellershoff, D. (1976): Literatura y principio de placer, Madrid, Ed. Guadarrama.

- Yubero, S. y Larrañaga, E. (2OIO): “El valor de la lectura en relación con el comportamiento lector. Un estudio sobre los hábitos lectores y el estilo de vida en niños”, Ocnos (6), Castilla-la Mancha, pp. 7-20. 